\title{
Clases naturales: una revisión crítica desde la causalidad y la complejidad
}

\author{
Natural Kinds: \\ a critical review from causality and complexity
}

\author{
ELIZABETH MARTÍNEZ BAUTISTA \\ IIF- Universidad Nacional Autónoma de México
}

Recibido: 8-XI-2013 Aceptado: 9-I-2014

\begin{abstract}
RESUMEN
Las clases naturales tienen un importante valor heurístico que no ha sido explorado a nivel epistémico. Una revisión crítica del concepto de "clase natural" sugerido por la teoría del Cúmulo de Propiedades Homeostáticas (HPC), desde la complejidad y la causalidad, muestra que es incompatible con las prácticas científicas, en especial las de la biología. Debido a los múltiples problemas que presenta en ese ámbito científico el concepto de 'clase natural' debería ser reconfigurado con el fin de estar acorde con las inferencias explicativas y predictivas de la biología. Esto tendría como consecuencia un mejor entendimiento filosófico de los procesos y entidades naturales a la vez que ayudaría a resolver algunos problemas filosóficos y científicos.

PALABRAS CLAVE

CLASE NATURAL, CAUSALIDAD, COMPLEJIDAD, PRACTICAS CIENTÍFICAS, ESPACIOS DE POSIBILIDAD EPISTÉMICOS
\end{abstract}

\begin{abstract}
Natural kinds have an important epistemic value that has not been studied due to the fact that the philosophical research has been focused on both semantics and logical aspects. I propose a critical review from causality and complexity to the concept of «natural kind» advanced by Homeostatic Properties Cluster Theory (HPC) in order to demonstrate that the kinds in the HPC style s are not supported by scientific practices such as biology. I think that a different concept of "natural kind" should be based on scientific practices in order to obtain a better philosophical understanding compared to HPC of both, natural processes and obtaining successful inferences. I hold that this epistemic approach provides us a new characterization of
\end{abstract}

(C) Contrastes. Revista Internacional de Filosofía, vol. XX-N ${ }^{\circ} 1$ (2015), pp. 135-152. ISSN: 1136-4076

Departamento de Filosofía, Universidad de Málaga, Facultad de Filosofía y Letras Campus de Teatinos, E-29071 Málaga (España) 
"natural kind" that will be useful as a unifying concept of natural phenomena, as well as for generating new spaces of epistemic possibility

KEY WORDS

NATURAL KINDS, CAUSALITY, COMPLEXITY, SCIENTIFIC PRACTICES, EPISTEMIC POSSIBILITY SPACES

\section{INTRODUCCIÓN}

EL PUNTO DE PARTIDA de una reflexión filosófica sobre las prácticas científicas reside en el análisis de las diversas herramientas epistémicas que construyen los científicos para explicar y predecir lo que sucede en el mundo natural. Tales construcciones epistémicas están basadas en criterios y supuestos específicos sobre lo que es legítimo reconocer como existente en cierto contexto de investigación.

Para explicar el éxito de las prácticas científicas, las clases naturales son importantes debido a su capacidad tanto heurística, como epistémica, para explorar las inferencias derivadas de la investigación. Por ello, en este trabajo, mi interés principal es encaminar la discusión sobre las clases naturales fuera de una metafísica esencialista y centrarla en las implicaciones epistémicas que se deriven de su uso como dispositivos heurísticos en la obtención de inferencias exitosas.

Aunque la noción de «clase natural» resulta problemática no debería ser abandonada, sino re-conceptualizada de modo tal, que sea compatible con la concepción científica de 'especie' La propuesta que presento no tiene como finalidad ofrecer una definición terminante de qué es una clase natural, sin embargo, el concepto es epistémicamente útil porque captura la intuición de que, sobre la base de la similitud y diferencia, es posible establecer una clase de objetos a la cual recurrir en las inducciones y explicaciones científicas de la biología. Esto tendría como como consecuencia la incursión en nuevos espacios de posibilidad epistémicos que permitan la solución de problemas filosóficos y científicos.

En lo que sigue, presento una breve descripción del estado actual de la discusión sobre una concepción mecanicista de «clase natural» ofrecida por Richard Boyd (1999), que ha servido como base a versiones actualizadas sobre las clases naturales (Wilson et al, 2007). También presento mis críticas a tal concepción de clase natural y finalmente sugiero la línea argumentativa que pudiera seguirse para futuras investigaciones sobre las clases naturales.

\section{UNA EXPLICACIÓN MECANICISTA SOBRE LAS CLASES}




\section{NATURALES: LA TEORÍA HPC (SUS ALCANCES Y LÍMITES)}

En la filosofía de la ciencia y del lenguaje, el concepto de «clase natural» ha estado asociado con otros conceptos igualmente problemáticos como, por ejemplo, el de «esencia». Esto se debe, en mi opinión, a que la aproximación filosófica al tema de las clases naturales ha estado dominada por perspectivas semánticas o metafísicas y se ha dejado de lado la perspectiva epistémica. La epistemología, que puede ser considerada como la multiplicidad de actividades asociadas con la investigación científica (Giere et al 2006), será en donde se centre mi discusión sobre las especies biológicas en tanto clases naturales.

Considero que el concepto de «clase natural» es intuitivo, ya que en primera instancia sería difícil rechazar la idea de que «las cosas que se parecen entre sí son de una misma clase» (Kripke, 1980). Pero los problemas sobre las clases naturales $(\mathrm{CN})$ surgen cuando, por un lado, se trata de dar criterios de pertenencia a una clase en particular, y por otro, se investiga qué beneficio epistémico pudiera tener el reconocimiento de tales entidades. Es en este último aspecto en donde las clases naturales tienen importancia, ya que son conjuntos de objetos con características específicas lo que permite hacer inferencias científicas exitosas sobre su naturaleza y sus relaciones.

La Teoría del Cúmulo de Propiedades Homeostáticas (HPC) propuesta por Richard Boyd (1999a), resultó exitosa como explicación filosófica sobre las $\mathrm{CN}$ al remplazar la noción tradicional de esencia por la de mecanismos homeostáticos. Un mecanismo homeostático puede ser considerado como un sistema que mantiene el estado estable de otro sistema que lo contiene. También sugirió que el reconocimiento de las clases naturales, en el contexto de las matrices disciplinarias o disciplinas científicas (Boyd, 1999a), contribuye al éxito inferencial de las prácticas científicas.

En la concepción de la teoría HPC, las clases naturales son un conjunto de individuos que pueden instanciar algunas, no necesariamente todas, las características de un cúmulo de propiedades homeostáticas. Esto sugiere, que en HPC, hay un reconocimiento de la imperfecta similitud entre los miembros de una clase, de allí que su criterio de pertenencia sea en términos de suficiencia más que de necesidad. Por otra parte, el conjunto de propiedades posee cierta estabilidad debido a mecanismos homeostáticos subyacentes los cuales son causalmente responsables de mantener su identidad (Boyd, 1999a).

Esta caracterización de clase natural de HPC puede aplicarse a cualquier clase, pero en especial a las especies biológicas. Agrupados sobre la base de lo que Boyd llama «características superficiales», los individuos de una especie biológica conforman una clase natural. La identidad de la clase está asegurada por mecanismos causales subyacentes como recombinación de genes, transcripción de genes, regímenes de selección similar y restricciones ontogenéticas 
comunes, entre otras (Boyd, 1999b). Estos mecanismos son los responsables de inducir la uniformidad observable de una especie biológica.

Aunque la teoría HPC reemplazó la debatible noción metafísica de esencia por el concepto científico de «mecanismo», no por ello ha quedado sin criticar. Los problemas que conlleva el proponer a los mecanismos homeostáticos como factores de individuación para una especie, resultan determinantes para descartar cualquier explicación sobre las CN en la línea argumentativa de HPC.

Como han mostrado varios autores, por ejemplo, Ereshefsky y Reydon (2013), Khalidi (2013), Ereshefsky (2010), Ereshefsky y Matthen (2005), Craver (2009), tanto los mecanismos como las características compartidas por los miembros de una clase son insuficientes para caracterizar una especie, ya que la función que desempeñan algunos mecanismos no siempre deriva en efectos uniformes observables. Esto es un problema para HPC porque las características observables son las que en última instancia determinan la pertenencia a una clase natural (Boyd, 1999a). Debido a estas críticas, la propuesta HPC ha sido actualizada por Wilson, Barker y Brigandt (2007) aunque también se han desarrollado líneas argumentativas al estilo HPC por Griffiths (1999), Okasha (2002), LaPorte (2004), Devitt (2008), Diéguez (2013), Rieppel (2013).

Las nuevas versiones al estilo HPC proponen a las propiedades relacionales como aquellos marcadores que permiten identificar a los miembros de una especie. De acuerdo con estas propuestas, las relaciones entre individuos o entre individuos y el ambiente, son determinantes para la pertenencia a un taxón. Por ejemplo, ser descendiente de un ancestro particular, pertenecer a una población determinada, ocupar un nicho particular (Okasha, 2002; LaPorte, 2004) son condiciones necesarias para determinar la pertenencia a un taxón.

Por otra parte, HPC ha tenido una gran aceptación en la filosofía de la ciencia debido a su aparente abandono de supuestos metafísicos y su pretensión de ser consistente con las prácticas científicas. Para HPC, el reconocimiento de las «clases naturales» tiene sentido a la luz de la tesis de la «acomodación» de las inferencias científicas de una matriz disciplinaria.

La tesis de la acomodación sugiere que los individuos clasificados bajo un término de clase natural comparten una estructura causal estable y este hecho explica por qué la referencia a tales clases ayuda a la convergencia de las clasificaciones científicas con la estructura causal del mundo. Es decir, el problema de la justificación del éxito de la explicación e inducción, dada cierta práctica científica, puede ser resuelto analizando la contribución que hacen las CN para lograr el éxito científico.

No obstante las virtudes epistémicas asociadas a esta explicación mecanicista sobre las CN, en mi opinión, como en la de otros autores (Ereshefsky y Reydon (2013), Khalidi (2013), Ereshefsky (2010), Ereshefsky y Matthen (2005), Craver (2009)), la teoría HPC no logra una descripción afortunada de 
los procesos externos como tampoco de los subyacentes que configuran la identidad de una especie. Tampoco es acorde con las prácticas taxonómicas de la biología y éstas últimas son importantes «porque son herramientas de inferencia y prueba» (Wilkins, 2012) de hipótesis sobre las especies biológicas. Además, HPC sólo explica parcialmente el éxito de las inferencias científicas.

Pese a que HPC no ofrece una explicación satisfactoria sobre las clases naturales, contiene otros aspectos que pueden ser rescatables. En tanto que los conceptos de «mecanismo», «propiedad relacional», «causalidad»y «homeostasis» son epistémicamente útiles para la descripción de entidades y procesos naturales, estos deberían de ser situados en una ontología acorde con la naturaleza dinámica de las especies biológicas y con la epistemología de las prácticas científicas taxonómicas actuales.

\section{ONTOLOGÍA Y EXPLICACIÓN}

A continuación desarrollo mis críticas a HPC, pero antes empezaré por definir al menos tres términos que considero importantes para un mejor entendimiento de mi argumentación: 'ontología', 'taxonomía' y 'práctica científica'.

Una ontología «es una representación de las clases de entidades que existen y de las relaciones entre ellas» (Gruber, 1998; Mabee, 2007; Love, 2008). Por ejemplo, en química, la tabla periódica constituye una ontología y en sistemática, los cladogramas son una ontología.

La ontología entendida en el sentido ya descrito está determinada por las prácticas científicas que pueden ser consideradas como modos o estilos de hacer ciencia (Martínez, 2010). Una práctica científica taxonómica bien pudiera ser la sistemática cladística (Hennig, 1960) o también la sistemática evolucionista (Mayr, 1959). Dependiendo de la práctica taxonómica, en una ontología se reconocerá o no, al grupo Reptilia como una clase (taxón) con valor epistémico. Esta es la manera como considero que la ontología es dependiente de los modos de hacer ciencia.

En una ontología, las entidades y sus relaciones deben ser situadas en un lugar común que permita representar los procesos naturales y generar inferencias exitosas en cierta práctica científica (Sarkar, 1998). El lugar común, en biología, son las taxonomías. Una taxonomía es una unidad representativa con poder explicativo bajo la cual se integran partes o aspectos de los organismos (Love, 2008).

Dado lo anterior, resulta epistémicamente importante una investigación sobre el tipo de ontología que asumen las teorías sobre las clases naturales en la línea argumentativa de HPC, la función que tienen las taxonomías y las clases naturales, en las prácticas científicas según esta propuesta y finalmente, será conveniente revisar si HPC es consistente con las matrices disciplinarias, como es su objetivo. También, evaluar si logra explicar satisfactoriamente la 
convergencia (tesis de la acomodación) entre la realidad natural descrita por una ontología y las prácticas científicas.

\section{1 DE LA ONTOLOGÍA DE LO LINEAL A LO COMPLEJO}

El tipo de entidades que reconoce HPC como significativamente epistémicas son individuos, clases de individuos y mecanismos. El tipo de relaciones que se establecen entre tales entidades son de tipo causal, relacional y homeostático. Hasta este punto, la ontología de HPC parece ser consistente con la de la biología «pero como casi todo en filosofía los problemas vienen cuando se miran los detalles» (Martínez, 2010).

En primer lugar revisaré el concepto de «causalidad». El tipo de causalidad no-lineal que las prácticas taxonómicas reconocen como determinante para la identidad de una especie, difiere de la causalidad lineal que HPC asume que es importante para establecer una clase natural.

En HPC, la causalidad es entendida como un factor que opera secuencialmente mediante un mecanismo homeostático como misma causa-mismo efecto (de ahí que se le llame lineal). La suposición de HPC es que en los individuos de una clase natural operan mecanismos homeostáticos que siempre los conducen al mismo estado final. Pero, en las prácticas taxonómicas actuales, el recurrir a la causalidad compleja, entendida como el hecho de que múltiples causas producen un efecto y varios efectos pueden tener una misma causa (causalidad no-lineal), es el mejor modo de explicar, tanto las regularidades, como la variabilidad fenotípica que muestran los organismos de una especie.

Para que los datos obtenidos de este tipo de causalidad compleja resulten significativos y tratables en la investigación científica, son linealizados con fines operacionales, mediante procesos estadísticos y modelación bioinformática. De esta manera, es posible establecer las causas más probables que dan origen a efectos observables al «utilizar grafos dirigidos para describir las relaciones causales entre las variables y regresión lineal para estimar el tamaño (coeficientes de trayectoria) de cada efecto» (Laland, 2011).

Lo anterior quiere decir al menos dos cosas importantes. Primero, que el compromiso epistémico que asumen las matrices disciplinarias (prácticas científicas) en sistemática, no es descubrir las causas esenciales de las regularidades observables sino que, dada cierta información, establecen una causa posible consistente con los datos disponibles. Segundo, que no obstante que los datos son ajustados linealmente con fines epistémicos para obtener filogenias, en la naturaleza, la complejidad causal es determinante en los procesos de conformación de una especie. El error de los recuentos de HPC en general, es tomar los datos «lineales» interpretados, como «verdaderos» en un sentido de correspondencia con la realidad. 
Por otra parte, las características observables de una especie son significativas en la práctica científica pero de manera muy distinta a la que HPC propone. En sistemática es innegable que las regularidades fenotípicas compartidas por los organismos sirven para identificar un taxón. Este tipo de práctica clasificatoria de los organismos en especies nos dice cómo reconocer un taxón, pero en tanto que sólo son un indicador de la clase de objeto que puede ser, las similitudes no responden a la pregunta filosófica sobre la justificación de por qué son como son y se comportan como lo hacen.

Dado que HPC está en la línea del tipo de explicaciones mecanicistas (Wimsatt, 1974; Salmon 1989; Glennan, 1996; Machamer, Darden \& Craver, 2000) responderá a la pregunta filosófica mediante una caracterización de los fenómenos naturales que capture la estructura causal del mundo y, por ello, recurre a los mecanismos homeostáticos. Sin embargo, su entendimiento de cómo funcionan éstos hace que su ontología se separe de la ontología científica.

Para mostrar que las críticas que he mencionado contra HPC tienen un referente en las prácticas de la biología, proporcionaré ejemplos de mecanismos genéticos del desarrollo y de procesos metabólicos homeostáticos. Estos ejemplos tienen por objeto mostrar que la función de los mecanismos, la homeostasis y la importancia de las características fenotípicas a nivel poblacional en una especie, es muy distinta de lo que HPC supone.

\section{II.2.LOS MECANISMOS REGULADORES DEL DESARROLLO DE LOS SERES VIVOS}

Los organismos que presentan simetría respecto a un plano sagital que divide el cuerpo en dos mitades idénticas, han sido llamados organismos bilaterales. Todos los organismos bilaterales poseen un mecanismo genético común que regula su desarrollo a lo largo de un eje antero-posterior. Tales mecanismos han sido llamados mecanismos Hox (Hueber \& Lonhman, 2008).

Las proteínas Hox especifican la diversidad morfológica mediante la activación o represión de la transcripción de ciertos genes (Carroll, 2005.) Por ejemplo, cuando la expresión del gen que codifica para la proteína HOX es suprimida, hay una pérdida de límites en los segmentos maxilares, en tanto que su restauración mantiene los límites segmentarios mandibulares (Longhman et.al., 2002). Esto puede hacernos suponer que los mecanismos Hox operan vía una causalidad lineal entre genotipo y fenotipo como HPC propone.

Sin embargo, no hay relaciones causales directas entre genes Hox y fenotipos, porque los mecanismos de regulación presentan un comportamiento complejo al momento de especificar las identidades de los segmentos en los organismos bilaterales. Para entender la complejidad funcional de los mecanismos Hox se debe comprender su función en un marco de redes regulatorias genéticas GRN (Hueber \& Lonhman, 2008). 
La operación de los mecanismos Hox sugiere que su función ontogenética involucra redes causales no lineales debido a la redundancia funcional genética. Cuando una función bioquímica es codificada por dos o más genes, originando que algunos de ellos contribuyan en modo equivalente a la realización de funciones que desempeñan otros, hablamos de redundancia funcional genética (Pearce S., 2004; Enns L.C., 2005).

Estas redes causales regulatorias son regiones de ADN que controlan las señales de la embriogénesis para la construcción de un «plan» de desarrollo corporal mediante procesos no-lineales que pueden ser considerados como interacciones moleculares de carácter estocástico. La reconstrucción de estas redes causalmente responsables de la expresión fenotípica de los sistemas biológicos, no sólo toma en cuenta al mecanismo genético, también depende de los contextos (células, tejidos, sistemas) que especifican las interacciones con los factores de transcripción Hox.

El punto que muestra este ejemplo es que de hecho, existen mecanismos que inducen la expresión fenotípica regular (similitudes) para distintos organismos, pero tales mecanismos no nos servirían como criterio para clasificar organismos en una misma clase porque no son únicos para un grupo específico con similitudes compartidas.

Siguiendo la línea argumentativa de HPC, que propone a los mecanismos homeostáticos como causalmente responsables de la uniformidad fenotípica de los individuos de una especie, las consecuencias taxonómicas serían las siguientes: o bien asignamos a todos los organismos bilaterales que comparten los mecanismos Hox en una misma especie, lo que contradice las prácticas taxonómicas, o bien se acepta que los mecanismos homeostáticos son insuficientes para explicar por qué los organismos son de cierta especie.

Así que, en este ejemplo de mecanismos homeostáticos que regulan la ontogenia, surgen dos problemas para HPC: por un lado la imposibilidad de determinar qué mecanismos son esenciales para establecer la pertenencia a un taxón y, por otro, si aceptan que los mecanismos funcionan en redes complejas aleatorias deberán abandonar la idea de mecanismos esenciales a una especie. Entonces, una ontología al estilo de HPC que reconozca a los mecanismos homeostáticos como esenciales a los organismos y cuya función de éstos sea causalmente lineal parece insuficiente para lograr una defensa exitosa de la tesis de la «acomodación» de las inferencias científicas. Aun así, como se discutirá más adelante, el problema de la «acomodación» no debe presuponer un descarte de la noción de especie ya que es posible cuantificar la extensión de «especie» sobre la base de otros factores, aparte de los mecanismos, la extensión de «especie». 


\section{3. LA HOMEOSTASIS ES ¿ESTABILIDAD?}

En la sección anterior he mencionado que los mecanismos homeostáticos que regulan la morfología de algunos organismos no constituyen procesos esenciales subyacentes a un grupo particular como para considerarlos la base que explica la «acomodación» entre teoría y mundo. Pero, ya que de hecho los individuos de una especie presentan regularidades y similitud morfológica ¿estas características se deben a una homeostasis en el sentido de HPC?

La noción de 'homeostasis' ha sido entendida de manera equivocada en la propuesta de HPC (DeLanda, 2002; Bechtel \& Abrahamsen, 2012). Desde que fuera usado por Walter Cannon (1929) para describir la constancia del ambiente interno de los organismos, el término es asociado con la capacidad de un organismo para mantener un estado de equilibrio con su ambiente.

Esta concepción de la homeostasis es adecuada para sistemas termodinámicos aislados experimentalmente en los cuales no hay intercambio de materia ni energía con su entorno (Lewis \& Randall, 1961), pero los organismos están lejos de presentar este tipo de equilibrio. Un sistema biológico, como una especie, presenta múltiples capacidades de reacción auto organizativa frente a presiones externas e internas. Se le llama «autoorganización»a la emergencia espontánea del orden en sistemas físicos y naturales (Kauffman, 1993).

La capacidad autónoma de los sistemas biológicos para re-organizarse, más que ser caracterizada como un estado de equilibrio, refiere a un tipo de estabilidad asintótica o aproximativa ya que nunca es alcanzada totalmente. Así que la homeostasis en los sistemas biológicos es en realidad un estado de tendencia al equilibrio (Bechtel \& Abrahamsen, 2012).

Hasta este punto, entender la homeostasis de manera distinta puede parecer sólo una diferencia conceptual sin embargo, la manera de caracterizar un proceso particular incide directamente en la configuración de una ontología y por ende, en las consecuencias epistémicas que pudieran derivarse de los conceptos, las entidades y sus relaciones utilizados en su descripción.

En términos de HPC, los mecanismos homeostáticos operan secuencialmente dando como resultado los mismos estados observables en todos los organismos de una clase. Puesto que el resultado, cada vez que opera el mecanismo, es una regularidad sobre la que se basan las explicaciones y predicciones científicas, pareciera que la «acomodación» es lograda como HPC supone.

Pero si la causalidad fuera secuencial, la interrupción de uno de los pasos en la secuencia tendría como consecuencia la pérdida del estado óptimo en un organismo. Sin embargo, los organismos ajustan sus funciones a los cambios, lo que quiere decir, que el tipo de causalidad asociada a la homeostasis no es de tipo secuencial como HPC supone y, por ende, la homeostasis también necesita ser entendida de manera distinta. 
Algunos mecanismos que regulan comportamientos repetitivos en los organismos funcionan con una organización no-secuencial entre sus partes y operaciones lo que permite, mas no garantiza, las regularidades observables (Bechtel \& Abrahamsen, 2012). Por ejemplo, en los mamíferos, un mecanismo ubicado en el núcleo supraquiasmático $(\mathrm{SCN})$ del hipotálamo controla los ritmos circadianos (cercanos a $24 \mathrm{~h}$ ). El mecanismo, un master clock, se ajusta a sí mismo de acuerdo a la entrada de la luz y sincroniza los relojes en los tejidos periféricos tales como el hígado y el riñón. Es sabido que, además de los SCN, los tejidos periféricos e incluso células cultivadas, tienen relojes autosustentables cuyo mecanismo molecular es muy similar al del reloj maestro (Mohawk et.al. 2012).

Los ajustes del mecanismo, lejos de constituir un estado estable del organismo, suponen un auto-restablecimiento de materia y energía, o actividad oscilatoria, entre la producción y degradación de sustratos bioquímicos. A esta actividad oscilatoria subyace un principio organizativo conocido como bucle de retroalimentación negativo que controla los picos y caídas (oscilaciones) en la concentración de la proteína PER, que después de ser sintetizada en el citoplasma, se mueve al núcleo para inhibir la transcripción de su propio mRNA. (Tyson, 2010).

El mecanismo regulatorio para la síntesis de la proteína PER, como se ha mencionado, contiene un bucle de re-alimentación negativa ralentizado. Los sistemas ralentizados que controlan comportamientos regulares de otros sistemas se les conocen también como sistemas hereditarios, sistemas de efecto retardado o sistemas $n$-dimensionales. La característica de estos sistemas homeostáticos es que la relación causa-efecto no solo depende del estado actual del sistema, sino de su historia (Ju, Chen, Kharitonov, 2003).

Para entender la diferencia entre el tipo de estabilidad que supone HPC para los mecanismos homeostáticos y el tipo de estabilidad que la investigación científica establece para estos, propongo entender cómo se describe su funcionamiento en la práctica científica.

Al describir los procesos involucrados en el funcionamiento de un mecanismo homeostático, se asume que este es un sistema dinámico sujeto a modelación. Un modelo matemático para describir un sistema dinámico es un conjunto de ecuaciones diferenciales ordinarias (ODEs) cuyas variables representan variables de estado y las ecuaciones caracterizan la evolución del sistema con respecto al tiempo (Ju et al 2003).

Para describir a los sistemas con efectos retardados, se utilizan ecuaciones diferenciales funcionales que tienen un gran número de soluciones. Esta característica nos dice que tales sistemas son sensibles a ciertas condiciones iniciales y como tal, por pequeño que sea el cambio en estas, los efectos serán sólo predecibles debido a su variabilidad. 
Pero para obtener una descripción del funcionamiento del bucle de retroalimentación se utilizan modelos cuyas descripciones corresponden a sistemas idealizados en donde se asume la uniformidad y estabilidad. Estas descripciones no deberían confundirse con los estados objetivos en la naturaleza, como parece hacerlo HPC. Los modelos nos «dicen hacia dónde se dirige un sistema mas no nos dicen lo que pasa en el sistema» (Tyson, 2001).

Por tales razones considero, al igual que otros autores (Machamer et. al. 2000, Bechtel \& Abrahamsen, 2012), que este tipo de trabajo experimental basado en simulaciones constituye un ejercicio de idealización y abstracción y puede ser un dispositivo heurístico para entender los complejos esquemas organizativos que se llevan a cabo, en tiempo real, en los organismos. En las simulaciones, el investigador tiende a considerar a los organismos como sistemas aislados bajo condiciones iniciales que determinan su estado final, pero en la naturaleza, los organismos son sometidos a distintas presiones ambientales lo que los obliga a ajustar su norma de reacción.

Por ejemplo, la manipulación de los elementos genéticos involucrados en los ciclos circadianos, como es el caso de las mutaciones en el gen per, han mostrado que el ciclo se alarga, acorta o se inhibe cuando estas son introducidas. Esto supone que la introducción de la variabilidad en un organismo resulta en un ajuste que estabiliza asintóticamente sus funciones (Knopka et al 1971).

Hasta ahora mi pretensión ha sido mostrar que los mecanismos homeostáticos de los sistemas biológicos funcionan de modo no-secuencial, ya que los organismos ajustan sus regularidades frente a presiones externas e internas a su metabolismo. Por otro lado, este ajuste supone condiciones cercanas al equilibrio, p.e los picos y caídas en las concentraciones de la proteína PER, cuya descripción obedece a sistemas dinámicos con efectos retardados.

Esta manera de entender la función de los mecanismos homeostáticos tiene como consecuencia una caracterización filosófica distinta a HPC sobre las entidades y procesos naturales, por ejemplo, el considerar tanto a los individuos como a las especies como sistemas dinámicos con estados finales abiertos. Es decir, las especies tienen configuraciones finales divergentes, debido a la variabilidad que presentan, y el estado final más probable es el que puede establecerse como una regularidad. Una caracterización de estas regularidades asintóticas de los organismos, que no es incompatible con su carácter estocástico, es considerarlas como propiedades emergentes del sistema sobre las que se basan las inferencias y explicaciones científicas.

Una propiedad emergente es una característica de un sistema que surge de sus interacciones causales complejas. Tales propiedades son caracterizables como irreducibles y emergentes, si y sólo si, no se pueden inferir a partir de la disposición de las partes de su sistema como tampoco si las propiedades se han «aislado» en otros sistemas más sencillos (Broad, 1919). 
Si bien las regularidades emergentes en distintas escalas pueden servir como base a las inferencias científicas debido a que muestran suficiente duración y estabilidad asintótica, estas no nos sirven para individuar una especie tal como HPC propone. El análisis estadístico a nivel de poblaciones de las regularidades fenotípicas emergentes de los organismos, involucra procesos de abstracción e idealización que muchas veces no tienen un correlato en la naturaleza. Por ejemplo, las capacidades de respuesta fenotípica poblacionales son medibles mediante tasas de cambio significativas, puesto que las cifras poblacionales, aun cuando tiendan a una regularidad, sólo oscilarán alrededor de un valor fijo.

Además, resulta importante considerar que «hay una tradición filosófica, a partir de Aristóteles, en la que simplemente similitudes observables son insuficientes para una explicación, por lo general tales similitudes son sólo suficientes para establecer 'que algo es así' mas no 'por qué es así'» (Ereshefsky \& Matthen, 2005). Lo anterior para mostrar que existen elementos, más allá de los mecanismos y las regularidades, que son determinantes en la conformación de una especie biológica y que deberían ser tomados en cuenta en una caracterización de clase natural.

\section{Clases naturales y espacios de Posibilidad epistémicos}

La descripción de las entidades y procesos naturales está enmarcada en los estilos actuales de hacer ciencia, lo que sugiere un cambio en la ontología y en los conceptos filosóficos. Una ontología para las clases naturales debería incluir a la diversidad, variabilidad y evolución, como factores constantes que configuran a una especie biológica, mientras que la epistemología para $\mathrm{CN}$, debería reconocer que esos factores, en la práctica científica, son considerados como datos lineales producto de la idealización y la abstracción.

La abstracción e idealización constituyen operaciones cognitivas del investigador que son determinantes en la construcción de espacios de posibilidad epistémicos o marcos de interpretación de la realidad natural. En tanto que son dominios epistémicos construidos, estos incluyen conceptos, estilos de razonamiento, taxonomías y ontologías.

Teniendo en cuenta estos procesos cognitivos de idealización, abstracción y aproximación, que generan conocimiento científico sobre la realidad natural, considero que se puede ofrecer una línea argumentativa que prepare el camino para la construcción de un concepto de «clase natural» distinto a HPC. Este concepto de $\mathrm{CN}$ deberá ser acorde con las prácticas y razonamientos científicos de modo tal, que permita la integración de las distintas conceptualizaciones de «especie» que hay en biología y que su definición, por ser tan general, no se comprometa con ningún concepto de estas en particular como para permitir la exploración de espacios de posibilidad epistémicos. 
Una concepción no-tradicional sobre las clases naturales las consideraría como dispositivos heurísticos que ayudan a explicar el éxito de las inferencias científicas, pero también como unidades representativas en donde colapsen taxonomías incompatibles sobre las especies biológicas (Love, 2008, Brigandt, 2009; Martínez, 2010). Esto permitiría la resolución de problemas científicos desde distintos puntos de vista, lo que está directamente relacionado con la exploración y construcción de espacios de posibilidad epistémicos.

Además, una definición alternativa de «clase natural», también tendría que capturar la variabilidad y evolución de los sistemas biológicos. Los teóricos modernos de la evolución, así como los biólogos del desarrollo, han propuesto nuevas formas de entender y explicar estos factores mediante nociones causales complejas relacionadas con el ambiente, la construcción de los nichos ecológicos y, en algunos casos, factores culturales (Pearl, 2000, Fuentes, 2011; Laland, 2011,).

Por otro lado, en tanto que una definición alternativa de «clase natural» haría alusión a la complejidad causal que muestran los sistemas biológicos en la naturaleza, su valor representacional, en un estilo particular de hacer ciencia, no sería neutral o indiferente a la realidad natural. En tanto que términos como «tigre», «oso» o «Reptilia» refieren a tipos $^{l}$ de entidades en la naturaleza, mantienen un vínculo con la realidad natural, sin embargo, al no disponer de información completa sobre los procesos que confieren identidad a una clase natural, se proponen criterios idealizados o abstractos para establecer su extensión. Si los criterios logran una representación aproximadamente correcta de esos varios procesos, entonces se obtienen inferencias explicativas y predictivas exitosas (Arroyo-Santos, 2011).

Por otra parte, considero que una consecuencia natural de una concepción distinta de clase natural, es que no constituiría una explicación reduccionista sobre las entidades y procesos naturales, como pudiera serlo una definición de clase natural, al estilo HPC, que reduce su caracterización a los mecanismos homeostáticos. Y esto, en mi opinión, es una ventaja epistémica fundamental sobre cualquier propuesta que intente seguir la línea argumentativa de Boyd (1999a) porque invita a la pluralidad de espacios de posibilidad epistémicos.

Los espacios de posibilidad epistémicos son importantes tanto en biología, como en filosofía, porque permiten pensar de qué modo puede ser utilizado un concepto y qué implicaciones tendría tal uso en la investigación científica. Ofrecen nuevos modos de pensar sobre los aspectos de la naturaleza, posibi-

1 En el contexto de la filosofía de la biología, y siguiendo la línea argumentativa de Alan Love (2009), consideraré a la tipología como «una forma de pensamiento o razonamiento [...] que incluye la representación y caracterización de un fenómeno natural agrupándolo y distinguiéndolo de acuerdo con diferentes características e ignorando variaciones particulares». 
litan el conocimiento de los sucesos naturales y cómo pudieran comportarse los sistemas biológicos bajo situaciones novedosas y, sobre todo, cómo llegar a generalidades desde pocos datos disponibles.

Así que, mientras un concepto alternativo de «clase natural» puede ser unificador de varios fenómenos, su función epistémica es promover la pluralidad de las interpretaciones de tales fenómenos al no comprometerse con una descripción única de estos como lo hace HPC al poner a los mecanismos homeostáticos como esenciales a una clase.

\section{CONCLUSIÓN: NuEVAS PERSPECTIVAS SOBRE LAS CLASES NATURALES}

A lo largo de este trabajo se ha mostrado que una caracterización de las clases naturales, que resulte epistémicamente útil para entender y explicar el éxito inductivo de las prácticas científicas, deberá ser compatible con éstas y redirigir la atención en los aspectos epistémicos en los que las clases naturales tienen un papel fundamental, como conceptos unificadores de fenómenos naturales diversos, pero también como generadores de nuevos espacios de posibilidad epistémica.

Estas características que propongo asociar al concepto de «clase natural» han sido poco exploradas, ya que generalmente, su caracterización se ha centrado en aspectos metafísicos o semánticos para dar cuenta de su valor epistémico. Centrar la atención en el valor heurístico que estas pudieran tener, más allá de la clásica justificación de las inferencias exitosas en la ciencia, constituye una manera distinta y prometedora de concebir las clases naturales.

Puesto que los fines científicos en biología están asociados hoy en día a un mejor entendimiento de los procesos de la naturaleza, el valor representacional unificador de varias perspectivas en una misma entidad (clase natural), puede resultar en una heurística que permita la exploración de recursos cognitivos para tratar con problemas clásicos como el de las especies o explicar la evolución y desarrollo de las especies desde distintas disciplinas con ontologías difíciles de conciliar.

Esta es la perspectiva y función por la que apuesta una concepción de clase natural como la que propongo y en esta línea de investigación, paralela a las prácticas científicas, considero que debería ir el tratamiento futuro sobre las clases naturales en filosofía.

\section{Agradecimientos}

Al Dr. Alfonso Arroyo-Santos, Dr. Antonio Diéguez Lucena y PhD. Federico Marulanda Rey quienes aportaron críticas y sugerencias significativas para la elaboración de este escrito. 


\section{BIBLIOGRAFÍA}

ARROYO-SANTOS, A. 2011: «Causalidad, epistemología y variables subrogadas en la construcción genética del mestizo mexicano», en Genes \& Mestizos, Ensayos sobre genómica y raza hoy.

BECHTEL, W. et.al., 2012: "Thinking dynamically about biological mechanisms: Networks of coupled oscillators, Foundations of Science", 1-17.

BOYD, R. 1999a: «Homeostasis, species, and higher taxa», en R. Wilson (ed.), Species: New Interdisciplinary Essays, 141-185.

BOYD, R. 1999b: «Kinds, complexity and multiple realization: comments on Millikan's 'Historical Kinds and the Special Sciences'», Philosophical Studies, 95: 67-98

BRIGANDT, I. 2009; "Homology: homeostatic property cluster kinds in systematics and evolution" (con Leandro C. S. Assis), Evolutionary Biology 36: 248-255.

BROAD, C.D.1925: The Mind and Its Place in Nature, Routledge \& Kegan Paul, London.

CANNON, W.B. 1929: Organization for physiological homeostasis, Physiol Rev, 9:(3) 399-431.

CARROL, S. 2005: «Evolution at Two Levels: On Genes and Form», PLoS Biology, Vol. 3, Iss. 7, e245.

CRAVER, C.F: 2009 Mechanisms and natural kinds, Philosophical Psychology 22: 575-594.

DELANDA, M. 2002: Intensive Science \& Virtual Philosophy, Continnum, 2002.

DEVITT, M. 2008: Resurrecting Biological Essentialism, Philosophy of Science 75, pp. 344-382

DIÉGUEZ, A. 2013: “Life as a Homeostatic Property Cluster”, Biological Theory, Volume 7, Issue 2.

ENNS, C. et.al. 2005: «Two callose synthases, GSL1 and GSL5, play an essential and redundant role in plant and pollen development and in fertility.» Plant Mol Biol 58(3).

ERESHEFSKY, M. et.al., 2005: «Taxonomy, Polymorphism, and History: An Introduction to Population Structure», Philosophy of Science, 72.

ERESHEFSKY, M. 2010: «What's Wrong with the New Biological Essentialism», Philosophy of Science, Vol. 77, No. 5.

ERESHEFSKY M. \& REYDON T.A.C. 2013: Scientific kinds. En prensa. Philosophical Studies.

FUENTES, A. 2011: Biological anthropology: concepts and connections, McGrawHill, NY.

GIERE, R. et.al. 2006: Understanding scientific reasoning, Wadsworth/Thomson Learning, Belmont.

GLENNAN, S. 1996: «Mechanisms and the Nature of Causation», Erkenntnis 44: 49-71.

GRIFFITHS, P. 1999: «Squaring the Circle: Natural Kinds with Historical Essences», en Species: New Interdisciplinary Studies, R. Wilson (ed.), MIT Press. 
GRUBER, T.R.1993: A translation approach to portable ontologies, Knowledge Acquisition 5, 199-220

HENNING, W. 1960: Elementos de una sistemática filogenética. EUDEBA, Buenos Aires.

HUEBER, D.S. et.al. 2008: «Shaping segments: Hox gene function in the genomic age», BioEssays 30:965-979.

GU, K, et.al., 2003: Stability of Time-Delay Systems, Springer.

KHALIDI, M. A: 2013 Natural categories and Human kinds, Cambridge University Press. New York.

KAUFFMAN, S. 1993: The Origins of Order: Self-Organization and Selection in Evolution, Oxford University Press, USA.

KNOPKA, B. et. al. 1971: Clock mutants of Droosophila melanogaster, Proceedings of the National Academy of Science (USA), 89.

LALAND, K. 2011: «Cause and Effect in Biology Revisited: Is Mayr's ProximateUltimate Dichotomy Still Useful?», Science 334.

LAPORTE, J. 2004: Natural Kinds and Conceptual Change, Cambridge University Press.

LEWIS, et.al. 1961: Thermodynamics, McGraw Hill, UC.

LOVE, A. 2008: Typology Reconfigured: From the Metaphysics of Essentialism to the Epistemology of Representation, Acta Biotheoretica, 57

LONGHMAN, I. et.al. 2002: «The Drosophila Hox gene Deformed sculpts head morphology via direct regulation of the apoptosis activator reaper», Cell 110:457-466.

KRIPKE, S. 1980: Naming and Necessity, Harvard University Press.

MABEE, P.M. et al. 2007: Phenotype ontologies: the bridge between genomics and evolution, Trends Ecol. Evol. Vol.30 No.10.

MACHAMER P.K. et.al. Thinking about mechanisms, Philosophy of Science 2000 67 (1):1-25.

MARTÍNEZ, S. 2010: La navaja de Ockham y la heterogeneidad de las representaciones: hacia una ontología de lo abstracto, azafea Revista de Filosofía, Vol. 12.

MAYR, E. 1963: Animal Species and Evolution. Harvard University Press

MOHAWK, J.A. et.al. 2012: Central and peripheral circadian clocks in mammals. Annu. Rev. Neurosci. 35.

OKASHA, S. 2002: Darwinian Metaphysics: Species and the Question of Essentialism, Synthese 131.

PEARCE, A.C. 2004: «Vav1 and vav3 have critical but redundant roles in mediating platelet activation by collagen», Biol Chem 279(52).

PEARL, J. 2001: Bayesianism and causality, or, why I am only a half-bayesian, en Fundations of Bayesianism, Kluwer Academic Publisher, Vol 24.

RIEPPEL, O. 2013: Biological individuals and natural kinds, Biological theory 7: 162-169.

SALMON. W, 1984: Scientific Explanation and the Causal Structure of the World, Princeton University Press.

SARKAR, S 1998: Genetics and reductionism. Cambridge University Press.

TYSON, J. 2001: Network dynamics and cell physiology, Nature Reviews Molecular 
Clases Naturales: una revisión crítica...

Cell Biology 2.

WILKINS J. et.al. 2012: The nature of classification, Kinds and Relationships in Natural Science, por publicarse.

WILSON, J. 2007: When Traditional Essentialism Fails, Philosophical Topics 35 (1-2)

WIMSATT, S. 1974: Reductive Explanation: A Functional Account, PSA, Vol. 1974

Elizabeth Martínez Bautista Doctorado en Filosofía de la Ciencia, Instituto de Investigaciones Filosóficas-UNAM

Línea de investigación: Filosofía de la ciencia y Filosofía del Lenguaje.

Dirección electrónica: marblesbaut@gmail.com 
